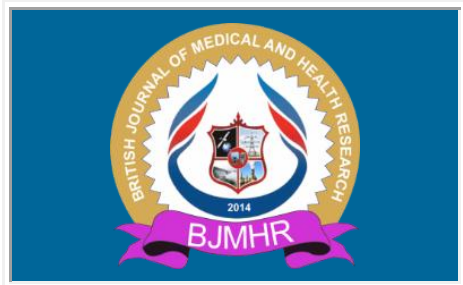

\title{
BJMHR
}

British Journal of Medical and Health Research Journal home page: www.bjmhr.com

\section{An Original Minimally Invasive Percutaneous Plate Osteosynthesis (Mippo) for Distal Fractures of the Tibia at the First Trimester of Pregnancy}

Adnane Lachkar

Department of Orthopedic Surgery. Centre Hospitalier Universitaire Mohamed-VI OujdaMorocco.

\section{ABSTRACT}

Surgical treatment of distal tibial fractures is challenging because of the limited soft tissue coverage and poor vascularity. Managing these orthopedic conditions in pregnant patient is a great challenge for the safety of both mother and fetus. Intra-operative fluoroscopy can be used just when appropriate. Minimally Invasive Plate Osteosynthesis (MIPO) technique is a method of fixation that reduces surgical trauma without exposure of fractured zone. By using an identical plate to the first one already placed percutaneously, plate on plate technique reduces radiation exposure, operating time, amount of surgical trauma and cost of treatment. The holes of the second plate are used to guide the mini skin incisions for accurate placement of screws. Here we present a case of distal tibial fracture at the first gestation trimester treated with MIPO using a plate on plate technique.

Keywords: MIPPO - tibial fractures - Pregnancy 


\section{INTRODUCTION}

Surgical treatment of distal tibial fractures is challenging because of the limited soft tissue coverage and poor vascularity. Anatomic reduction must be assured to obtain early union of fracture and functional return of activity. However, managing these orthopedic conditions in pregnant patient is a great challenge for the safety of both mother and fetus. Surgical considerations must be made based on physiologic changes during pregnancy, recommendations for monitoring and risks of radiation. Intra-operative fluoroscopy can be used just when appropriate. Regional anesthesia provides less overall drug exposure to the fetus and less variability in fetal heart rate. Lateral decubitus positioning is optimal to prevent hypotension due to compression of inferior vena cava. Also, there is an expanded risk for deep venous thrombosis (DVT) due to lower physiological hypercoagulable status. Treatment modalities of distal tibial fractures vary from closed reduction with cast application to open reduction and internal or external fixation passing by internal fixation with Minimally Invasive Plate Osteosynthesis (MIPO) technique. MIPO is a method of fixation that gains ample popularity; it reduces surgical trauma without exposure of fractured zone, it also limits soft tissue dissection, reduces wound related complications, preserves osteogenic fracture hematoma and improves union rates [1]. However the technique of screw placement uses too much fluoroscopic controls which are not always appropriate especially during pregnancy. By using an identical plate to the first one already placed percutaneously, plate on plate technique reduces radiation exposure, operating time, amount of surgical trauma and cost of treatment [1]. Holes of the second plate are used to guide mini skin incisions for accurate placement of screws. Here we present a case with distal tibial fracture at the end of first gestation trimester treated with MIPO using a plate on plate technique.

\section{MATERIAL AND METHOD}

A 29-year-old pregnant woman (11th week) presented to emergency department following an accidental fall down stairs. There was no history of any medical disorder. Upon examination, there was no evidence of neurovascular deficit or open wounds. Radiographic imaging showed a distal fracture of the right tibia classified as 42-A1-c according to AO/OTA Classification of Fractures and Dislocations (Arbeitsgemeinschaf fur Osteosynthesefragen (AO) and the American Orthopedic Trauma Association (OTA)) (figure 01.A). Following orthopedic examination, the obstetrician evaluated both fetus and mother; there were no restraints for surgical treatment. The patient was brought to operating room after stabilization. Spinal anesthesia was applied and a lead apron was placed over the abdomen to minimize fetus radiation doses. The fracture was reduced using manual traction and reduction was confirmed 
under image intensifier fluoroscopy. The joint line was marked with a needle of $5 \mathrm{ml}$ syringe and a small approximately $2 \mathrm{~cm}$ incision was made over medial malleolus for passing the plate (figure 01.B). A subcutaneous extra-periosteal tunnel was created using blunt end of the plate; it was then temporarily fixed to bone using $\mathrm{K}$ wires by passing them through the proximal and distal holes. An identical plate having the same number of holes (10 holes) was put over skin by passing it through the same $\mathrm{K}$ wires used for first plate fixation. The holes of the plate over skin were used for giving small incisions and passing screws; thus reducing amount of operation time, surgical trauma and radiation exposure. The plate was then fixed to bone using non-locking screws proximally via stab incisions and distally via previous oblique incision made for passage of plate. Wound was closed and limb was immobilized postoperatively in a removable splint. The patient was administered cold application, analgesics, and 4000 IU/day enoxaparin for prophylaxis. Postoperatively partial weight bearing was allowed at 6 weeks and full weight bearing at 12 weeks. The fetus and mother were evaluated serially by the obstetrician. At the 40th week, she had a healthy newborn via vaginal delivery.

\section{RESULTS AND DISCUSSION}

Up to $8 \%$ of pregnancies are affected by trauma [2]; it is the primary cause of non-obstetric maternal death. Falls and motor vehicle crashes are the most common mechanisms of injury [2]. The operative treatment of closed distal tibial fractures in the first trimester of pregnancy remains controversial. If the conservative treatment for this type of fracture is decided, the pregnant is using crutches and mobilization will be decreased making pregnancy so uncomfortable for the mother. When surgery is needed physiological changes that occur in pregnancy have implications for administration of anesthesia and peri-operative management. Typical first-line medications such as first- or second-generation cephalosporins, routinely used for orthopedic patients as perioperative antibiotics, are category-B agents and considered relatively safe in all trimesters (Table 01). Anesthetics may affect both the fetus and the mother; organogenesis occurs in the first trimester, and even if there are no anesthetic agents shown to be teratogenic, surgical procedures are often delayed until the second trimester [3]. However, it has been reported in the literature that the fracture union is accelerated because of the changed hormonal status during pregnancy [4] and a surgery planned later will be technically difficult in this case. There is no conclusive evidence that the technique of anesthesia, general or regional, has an impact on pregnancy outcome or that either type is safer than the other [2]. Although, in lower-extremity surgeries, spinal or epidural anesthesia is associated with lower drug exposure and has more benefit of providing superior postoperative pain control. While reviewing the literature, we found just two reports on surgical management of a tibial fracture during pregnancy $[5,6]$; the few other reports were all on conservative treatment. However, 
pregnancy is a known hypercoagulable state and, joined with the decreased mobility because of the tibial injury, anticoagulation is usually required. Warfarin is absolutely contraindicated and this drug should never be used during pregnancy. Low-molecular-weight heparins or unfractionated heparin at prophylactic doses are used for anticoagulation during pregnancy [7].

Table 1: Pregnancy and categories of antibiotics [2]

\begin{tabular}{ll}
\hline Antibiotics & Pregnancy category \\
\hline Cefazolin & B \\
Ceftazidime & B \\
Cephalexin & B \\
Clindamycin & B \\
Daptomycin & B \\
Gentamicin & D \\
Linezolid & C \\
Sulfamethoxazol & D \\
e-trimethoprim & \\
Tetracycline & D \\
Tobramycin & D \\
Vancomycin & C \\
\hline
\end{tabular}

Category B: agents relatively safe.

Category $\mathrm{C}$ : agents that may induce risk to the fetus

Category D: agents that should be avoided.

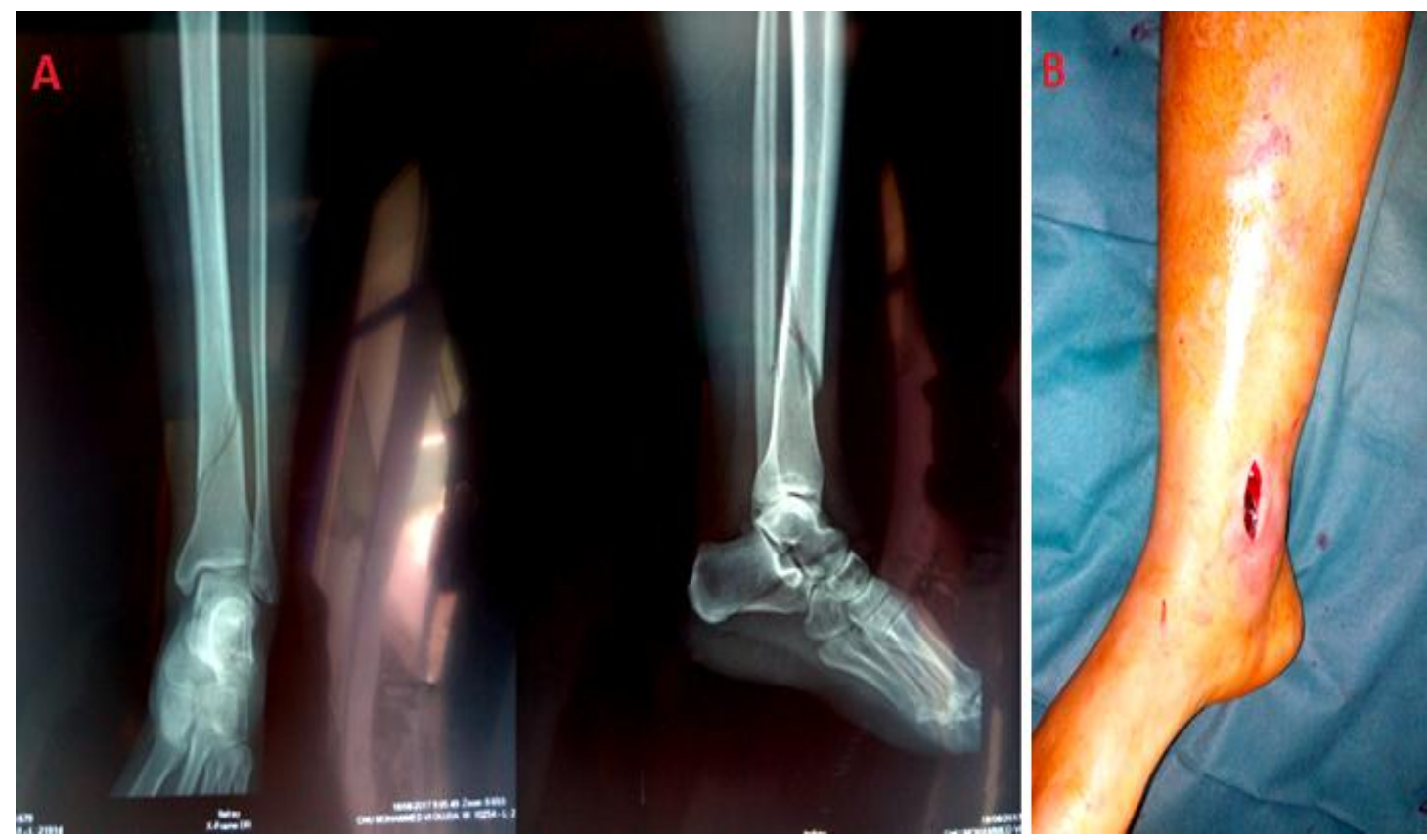

Figure 1: A. X-ray showing leg fracture. B. Perioperative photography showing position of stab incision

Radiation exposure affects the fetus more during the first trimester since development of central nervous system (CNS) is faster [7]. At the third trimester of gestation the CNS becomes more resistant to radiation but cumulative radiation effects are still significant. Fluoroscopy may 
exceed the teratogenic threshold. At 10 to 18 weeks' gestation, there is an increased risk of developmental delay or spontaneous abortion with exposure of greater than $100 \mathrm{mGy}$; the effects on a fetus at this gestational age are less clear at radiation doses of 50 to $100 \mathrm{mGy}$ [7]. The masse of radiation exposure from perioperative fluoroscopy is highly important using the classical percutaneous techniques; putting the screws with merely stab incision is possible but uses maximum fluoroscopy. With plate on plate technique, manual traction facilitates reduction of tibia in medio-lateral plane and this can be controlled by the image intensifier, then fracture reduction in antero-posterior plane and position of inserted plate is controlled in lateral projection; the reduction is then maintained with clamp. The use of a similar plate with same number of holes is of great help to locate the position of stab incision without radiation exposure [8]. Using plate on plate method is an inexpensive and easy surgical technique to decrease the radiation exposure and the operative time without increasing cost of surgical procedure [7]. Moreover, this technique preserves most of osseous vascularity and fracture hematoma and thus provides for a more biological repair [9]; it is also advantageous for small skin incisions, for limited soft tissue dissection and minimal hardware application.

\section{CONCLUSION}

Surgery can be planned only after obstetric examination with ultrasonography and a complete evaluation of the fetus. The surgical treatment chosen should especially consider the comfort of the mother and newborn. Thus, fracture management in a pregnant should be multidisciplinary, including orthopedic surgeon, anesthetist, obstetrician and neonatologist. The MIPO with the plate on plate technique offers practical and simple method to manage distal tibial fractures; it reduces radiation exposure, cost and operating time.

\section{REFERENCES}

1. Muzaffar N, Bhat R, Yasin M. Plate on plate technique of minimally invasive percutaneous plate osteosynthesis in distal tibial fractures, an easy and inexpensive method of fracture fixation. Arch Trauma Res. 2014 Sep 2; 3(3):e18325. doi: 10.5812/atr. 18325

2. Matthews LJ, McConda DB, Lalli TA, Daffner SD. Orthostetrics: Management of Orthopedic Conditions in the Pregnant Patient. Orthopedics. 2015 Oct;38(10):e874-80. doi: 10.3928/01477447-20151002-53.

3. Kuckowski KM. Nonobstetric surgery during pregnancy: what are the risks of anesthesia? Obstetrical \& Gynecological Survey, vol. 59, no. 1, pp. 52-56, 2004. doi: 10.1097/01.OGX.0000103191.73078.5F 
4. Ahmad MA, Kuhanendran D, Kamande IW, Charalambides C. Accelerated tibial fracture union in the third trimester of pregnancy:a case report. J Med Case Rep. 2008;2(1):44. doi: 10.1186/1752-1947-2-44.

5. Bozkurt C, Sarikaya B. A surgical opinion in a 36-week pregnant with tibia fracture: Intramedullary nailing. Case Rep Orthop 2016; 2016:4. doi: 10.1155/2016/1454835.

6. Ferguson M, Brand C, Lowe C, Gabbe B, Dowrick A, Hart M, Richardson M. Outcomes of isolated tibial shaft fractures treated at level 1 trauma centres. Injury. 2008; 39:187-195. doi: 10.1016/j.injury.2007.03.012.

7. Matthew LJ, McConda DB, Lalli TAJ, Daffner SD. Orthostetrics: management of orthopedic conditions in the pregnant patient. Orthopedics. 2015;38(10):874-80. doi: $10.3928 / 01477447-20151002-53$.

8. Güven M, Unay K, Cakici H, Ozturan EK, Ozkan NK (2008) A newv screw Wxation technique for minimally invasive percutaneous plate osteosynthesis. Acta Orthop Belg 74(6):846-850. doi: 10.1007/s11751-013-0164-9.

9. Paluvadi SV, Lal H, Mittal D, Vidyarthi K. Management of fractures of the distal third tibia by minimally invasive plate osteosynthesis - A prospective series of 50 patients. J Clin Orthop Trauma. 2014;5(3):129-136. doi:10.1016/j.jcot.2014.07.0100.

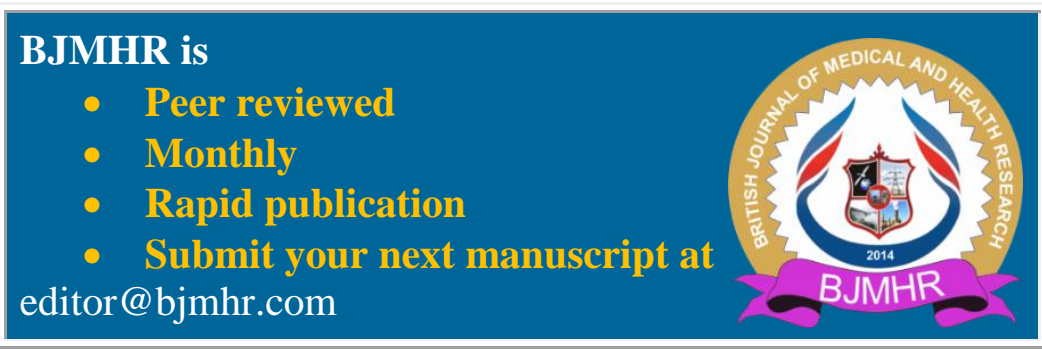

